SOGANG-HEP 230/97

SNUTP 98-014

\title{
Non-Abelian Ramond-Neveu-Schwarz String Theory
}

\author{
Hyun Seok Yang, Inbo Kim, and Bum-Hoon Lee \\ Department of Physics, Sogang University, Seoul 121-742, Korea
}

\begin{abstract}
We construct a locally supersymmetric worldsheet formulation of a nonAbelian Ramond-Neveu-Schwarz (NARNS) string theory where the string coordinates are noncommuting matrices in a group $U(N)$. This is described by the two dimensional supergravity coupled to supersymmetric Yang-Mills fields and adjoint matters in the gauge group $U(N)$. We show that our NARNS string theory has a free string limit where it becomes N-copies of usual RNS string which can be described by the orbifold conformal field theory corresponding to the covariant worldsheet version of the Matrix string theory of Dijkgraaf, Verlinde and Verlinde. In the weak coupling limit, i.e. $g_{s} \rightarrow 0$ where $g_{s}$ is the coupling constant of our theory related with the Yang-Mills coupling as $g_{Y M}^{-2}=\alpha^{\prime} g_{s}^{2}$, a new additional dimension appears in the string spectrum and it can be speculatively interpreted as the compactified eleven dimensional coordinate whose dynamics is given by an orbifold $\mathrm{O}(\mathrm{N})$ sigma model.
\end{abstract}

Keywords: Non-Abelian Ramond-Neveu-Schwarz string theory, Matrix string theory, supergravity, super-Yang-Mills, conformal field theory.

PACS numbers: 11.25.-w, 12.60.J

Typeset using REVTEX 


\section{INTRODUCTION AND MOTIVATION}

The nonperturbative formulation of string theory needs a mysterious eleven dimensional M-theory [1,2], which is a strong coupling limit of type IIA superstring theory and that its low energy limit is eleven dimensional supergravity which has membrane and M5-brane as fundamental degree of freedom as well as graviton. Though one is lacking an intrinsic definition of M-theory in terms of its underlying degrees of freedom, its mere existence led to many powerful predictions or simplifications of superstring duality [3]. Major step forward was taken by Banks, Fischler, Shenker and Susskind (BFSS) whose conjecture is that Mtheory quantum dynamics in the infinite momentum frame (IMF) is described by $U(N)$ supersymmetric Yang-Mills (SYM) quantum mechanics «4. One of the remarkable pictures of the BFSS matrix theory is that the spacetime coordinates live in a linear space of matrices which define so-called noncommutative spacetime geometry [5]. The classical commutative geometry is only sensible concept in a long distance regime. The matrix theory provides a natural and simple mechanism for the appearance of a noncommutative geometry at short distances [6].

It has been shown that the BFSS matrix theory compactified on a tiny circle, Matrix string theory (MST), provides a nonperturbative definition of the weakly coupled type IIA string theory [7],8]. The string coordinates of the MST are also matrices taking values in the non-Abelian gauge group although they become usual commutative C-numbers in the weak coupling limit which corresponds to the zero size limit of the compactified circle. The beautiful picture arises in the MST. It has a description of the Hilbert space of second quantized string theory [8]. The second quantized string is due to the residual discrete Weyl symmetry of the gauge group acting on the matrix elements within the Cartan subalgebra. In the BFSS matrix theory, this Weyl symmetry gives the conventional spin statistics on the states of the D0-brane Fock space [4].

Our motivation to construct a non-Abelian Ramond-Neveu-Schwarz (NARNS) string theory where the string coordinates are noncommuting matrices comes from this new picture, 
"noncommutative spacetime geometry". We think that there must exist a Lorentz invariant worldsheet formulation of MST which is a Green-Schwarz [9] light-cone formulation. What is the NARNS string theory and how the NARNS string theory should be constructed? It is a generalization of the usual RNS string theory [10 in the way that the string coordinates are noncommuting matrices in a group $G$, which depends on the worldsheet coordinate. Therefore the NARNS string theory is the two dimensional (2D) supergravity theory coupled to SYM fields and adjoint matters (string coordinates) in the gauge group $G$.

Recently the noncommutative spacetime picture of string theory appeared in an interesting way as the form of spacetime uncertainty relation by Yoneya and Li [11]. They argue that the spacetime uncertainty relation of the form $\Delta X \Delta T \geq \alpha^{\prime}$ for the observability of the distances with respect to time, $\Delta T$ and space, $\Delta X$, is universally valid in string theory including nonperturbative objects, D-branes [12] and this relation can be derived as a direct consequence of the worldsheet conformal invariance. It implies that the fumdamental constant $\alpha^{\prime}$ of Nature representing the string size has a fundamental significance as the constant $c$ (Lorentz covariance) and $\hbar$ (quantum mechanics). If we innocently accept their argument, the spacetime probed by string should be a noncommutative object for short distances compared to the string scale $l_{s} \equiv \sqrt{\alpha^{\prime}}$. Only for $\alpha^{\prime} \rightarrow 0$ limit, the classical geometry appears. This is an another motivation for our NARNS string theory.

The organization of this paper is as follows. In Sec.II, we formulate the superspace of 2D supergravity coupled to SYM fields for the purpose of constructing a locally supersymmetric worldsheet formulation of the NARNS string theory described above. Our superspace formulation of $N=1 \mathrm{SYM}$ theory coupled to $2 \mathrm{D}$ supergravity is a new one up to our knowledge. In Sec.III, we explicitly construct the NARNS string theory and show that it has a free string limit where it becomes N-copies of usual RNS string. And we observe that, in the weak coupling limit, i.e. $g_{s} \rightarrow 0$ where $g_{s}$ is the coupling constant of our theory related with the Yang-Mills coupling as $g_{Y M}^{-2}=\alpha^{\prime} g_{s}^{2}$, a new additional dimension appears in the string spectrum and it can be speculatively interpreted as the compactified eleven dimensional coordinate. In Sec.IV, we argue that the NARNS string theory is described by 
the orbifold conformal field theory (CFT) [13], essentially second quantized string theory, contrary to the ordinary RNS string which has a first quantized description. In the $g_{s} \ll 1$ limit with fixed $\alpha^{\prime}$, the SYM part must be considered. Nevertheless, the full superconformal symmetry of the NARNS string theory is preserved in a particular configuration. In the limit, the additional degree of freedom interpreted as compactified eleven dimension in this paper is interestingly described by the orbifold $\mathrm{O}(\mathrm{N})$ sigma model predicting that the size of this dimension increases in the ultraviolet limit and decreases in the infrared limit [14]. In Sec.V, we discuss many aspects of NARNS string theory. In Appendices, our conventions and some identities are listed and the details of the superspace formulation of 2D SYM theory coupled to supergravity are presented.

\section{2D SUPERGRAVITY COUPLED TO SUPER-YANG-MILLS THEORY}

In this section, we will formulate the superspace of $2 \mathrm{D}$ supergravity coupled to SYM fields for the purpose of constructing a locally supersymmetric worldsheet formulation of a NARNS string theory where the string coordinates are noncommuting matrices in a compact

Lie group $G$. We will closely follow the Wess and Bagger [15 on the superspace and Howe [16] on the 2D supergravity.

The 2D superspace has two kinds of supercoordinate indices, a curved index $M=$ $(m, n ; \mu, \nu)$ and a tangent index $A=(a, b ; \alpha, \beta)$. The coordinates of superspace, $z^{M}=$ $\left(\sigma^{m}, \theta^{\mu}\right)$, obey the following multiplication law:

$$
z^{M} z^{N}=(-)^{m n} z^{N} z^{M}
$$

At each point in superspace the one-form basis $E^{A}(z)$ define a local reference frame:

$$
E^{A}=d z^{M} E_{M}^{A}(z)
$$

where superzweibein $E_{M}^{A}(z)$ is an arbitrary invertible superfield,

$$
\begin{aligned}
& E_{M}^{A}(z) E_{A}^{N}(z)=\delta_{M}^{N} \\
& E_{A}^{M}(z) E_{M}^{B}(z)=\delta_{A}^{B} .
\end{aligned}
$$


The exterior derivative may be written in terms of the differential operator in the local frame (2.2)

$$
d=d z^{M} \frac{\partial}{\partial z^{M}}=E^{A} D_{A}
$$

where

$$
D_{A}=E_{A}^{N} \frac{\partial}{\partial z^{N}}
$$

The tangent frame $E^{A}(z)$ is locally Lorentz covariant:

$$
\delta E^{A}(Z)=E^{B} L_{B}^{A}(z)
$$

where the Lorentz generators $L_{B}{ }^{A}$ have two irreducible components:

$$
\begin{aligned}
& L_{B}{ }^{A}(z)=L(z) \mathcal{E}_{B}{ }^{A} \\
& \mathcal{E}_{B}{ }^{A}=\left(\begin{array}{cc}
-\epsilon_{a}{ }^{b} & 0 \\
0 & \frac{1}{2}\left(\gamma_{5}\right)_{\alpha}{ }^{\beta}
\end{array}\right) .
\end{aligned}
$$

To define covariant derivative on 2D Lorentz group we must introduce a connection form

$$
\Omega_{B}{ }^{A}=\Omega_{\mathcal{E}_{B}}{ }^{A}=d z^{M} \Omega_{M, B}{ }^{A},
$$

transforming as follows under the Lorentz group:

$$
\delta \Omega=-d L
$$

The connections allow us the covariant derivatives, for example, for a one-form $V$,

$$
\mathcal{D} V=d V+V \Omega
$$

Then the torsion two-form, $T^{A}$, is defined as the covariant derivative of the vielbein and the curvature two-form, $R_{A}{ }^{B}$, in terms of the connection:

$$
\begin{aligned}
T^{A} & =\mathcal{D} E^{A}=d E^{A}+E^{B} \Omega_{B}{ }^{A} \\
& =\frac{1}{2} E^{C} E^{B} T_{B C}^{A}, \\
R_{A}{ }^{B} & =d \Omega_{A}^{B}+\Omega_{A}{ }^{C} \Omega_{C}{ }^{B} \\
& =\frac{1}{2} E^{D} E^{C} R_{C D, A}{ }^{B} .
\end{aligned}
$$


They satisfy the Bianchi identies,

$$
\begin{aligned}
& \mathcal{D} T^{A}=E^{B} R_{B}{ }^{A} \\
& \mathcal{D} R_{B}{ }^{A}=0 .
\end{aligned}
$$

The 2D Lorentz group structure, Eq.(2.6), allows us to make the simplication

$$
R_{A}^{B}=F \mathcal{E}_{A}^{B} ; \quad F=d \Omega .
$$

In order to formulate $2 \mathrm{D}$ supergravity coupled to $N=1 \mathrm{SYM}$ theory, we must introduce a Lie algebra valued one-form:

$$
\begin{aligned}
& A=d z^{M} A_{M}=E^{B} A_{B}, \\
& A_{B}=A_{B}^{r} T^{r}, \quad r=1, \cdots, \operatorname{dim} G,
\end{aligned}
$$

where the matrices $T$ are the hermitean generators of the structure group $G$. Under a local structure group represented by $U=e^{i X}$ where the gauge parameter $X$ is a scalar superfield, the gauge connections transform as

$$
A^{\prime}=U^{-1} A U-i U^{-1} d U
$$

and we can define gauge covariant derivatives as before

$$
\nabla \phi=d \phi-i g_{Y M}(\phi A \pm A \phi)
$$

for a superfield $\phi$ in the adjoint representation of the group $G$. Here + is for odd form $\phi$ and - for even form. The curvature two-form tensor which can be constructed from the connection and its derivatives is defined as follows

$$
\begin{aligned}
F & =\frac{1}{2} d z^{M} d z^{N} F_{N M}=\frac{1}{2} E^{A} E^{B} F_{B A} \\
& =d A-i g_{Y M} A^{2}
\end{aligned}
$$

which covariantly transforms under the local structure group

$$
F^{\prime}=U^{-1} F U
$$


The curvature tensor, "field strength", may be then read as the component form

$$
F_{B C}=\mathcal{D}_{B} A_{C}-(-)^{b c} \mathcal{D}_{C} A_{B}+i g_{Y M}\left(A_{B} A_{C}-(-)^{b c} A_{C} A_{B}\right)+T_{B C}^{D} A_{D}
$$

As in the Eq.(2.12), the field strengths introduced above similary satisfy Bianchi identities by virtue of their definition in terms of 'potentials':

$$
\nabla F=d F-i g_{Y M}(F A-A F)=0
$$

in component form, which are

$$
\Delta_{[A} F_{B C\}}+T_{[A B}^{\mid D} F_{D \mid C\}}=0
$$

where [ $\}$ represents graded antisymmetrization and the derivatives $\Delta_{A} F_{B C}=\nabla_{A} F_{B C}-$ $\Omega_{A, B}{ }^{D} F_{D C}-\Omega_{A, C}{ }^{D} F_{B D}$ are the gauge and superspace covariant derivatives.

As the four dimensional case, we take the proper constraints on supertorsion to reduce the number of component fields, which must be Lorentz covariant, gauge covariant, and supersymmetric and should not restrict the $\sigma$-dependence of the component fields [15]:

$$
T_{\alpha \beta}^{a}=2 i\left(\gamma^{a}\right)_{\alpha \beta}, \quad T_{\beta \gamma}^{\alpha}=T_{b c}^{a}=0
$$

One can solve the Bianchi identities (2.12) subject to the constraints (2.21) and find that all the components of the curvature and the torsion can be expressed in terms of one scalar superfield $S$ [16]. Thus the supergeometry can be determined by the Bianchi identities and the torsion constraints.

In order to eliminate the gauge degrees of freedom coming from the superreparameterization and local Lorentz transformation, we impose the proper conditions on the superzweibein and the connection, so-called, Wess-Zumino gauge [15]. The component fields can then be derived from the superfield language in terms of their $\theta$ expansions [16];

\footnotetext{
${ }^{1}$ In fact, it can be showed that, for the choice of the tangent space group made here, the second Bianchi identity is derived from the first through its covariant derivatives, so that it is sufficient only to solve the first one.
} 


$$
\begin{aligned}
& E_{m}{ }^{a}=e_{m}{ }^{a}+i \bar{\theta} \gamma^{a} \chi_{m}+\frac{i}{4} \bar{\theta} \theta e_{m}{ }^{a} A, \\
& E_{m}{ }^{\alpha}=\frac{1}{2} \chi_{m}{ }^{\alpha}+\frac{1}{2} \theta^{\mu}\left(\gamma_{5}\right)_{\mu}{ }^{\alpha} \omega_{m}-\frac{1}{4} \theta^{\mu}\left(\gamma_{m}\right)_{\mu}{ }^{\alpha} A+\frac{i}{2} \bar{\theta} \theta\left(-\frac{3}{8} \chi_{m}{ }^{\alpha} A+\frac{i}{4}\left(\gamma_{m}\right)^{\alpha \beta} \psi_{\beta}\right), \\
& E_{\mu}{ }^{a}=i \theta^{\lambda}\left(\gamma^{a}\right)_{\lambda \mu}, \\
& E_{\mu}{ }^{\alpha}=\delta_{\mu}{ }^{\alpha}\left(1-\frac{i}{8} \bar{\theta} \theta A\right),
\end{aligned}
$$

and

$$
\begin{aligned}
& \Omega_{m}=\omega_{m}-\frac{i}{2} \bar{\theta} \gamma_{5} \chi_{m} A-\frac{1}{2} \bar{\theta} \gamma_{5} \gamma_{m} \psi-\frac{i}{4} \bar{\theta} \theta\left(\omega_{m} A+e \epsilon_{m}{ }^{n} \partial_{n} A+\frac{1}{2} \bar{\chi}_{n} \gamma_{5} \gamma_{m} \gamma^{n} \psi\right), \\
& \Omega_{\mu}=-\frac{i}{2} \theta^{\lambda}\left(\gamma_{5}\right)_{\lambda \mu} A, \\
& \omega_{m}=\frac{1}{e} e_{m a} \epsilon^{n l} \partial_{n} e_{l}{ }^{a}+\frac{i}{2} \bar{\chi}_{m} \gamma_{5} \gamma^{n} \chi_{n}, \\
& \psi=\frac{2 i}{e} \epsilon^{m n} \gamma_{5}\left(\partial_{m} \chi_{n}-\frac{1}{2} \omega_{m} \gamma_{5} \chi_{n}\right)-\frac{i}{2} \gamma^{m} \chi_{m} A .
\end{aligned}
$$

Here $A$ and $\psi$ are the first and second components of the curvature superfield $S$,

$$
\begin{aligned}
& S=A+\bar{\theta} \psi+\frac{i}{2} \bar{\theta} \theta C \\
& C=-\frac{2}{e} \epsilon^{m n} \partial_{m} \omega_{n}-\frac{i}{4 e} \epsilon^{m n} \bar{\chi}_{m} \gamma_{5} \chi_{n} A-\frac{1}{2} \bar{\chi}_{m} \gamma^{m} \psi-\frac{1}{2} A^{2},
\end{aligned}
$$

and $e=\left|\operatorname{det} e_{m}{ }^{a}\right|$. The superdeterminant must be introduced to construct invariant actions from superfields and can be calculated from superzweibeins, Eq.(2.22)

$$
E=\operatorname{sdet} E_{M}{ }^{A}=e\left(1+\frac{i}{2} \bar{\theta} \gamma^{m} \chi_{m}+\frac{i}{4} \bar{\theta} \theta A-\frac{1}{8} \bar{\theta} \theta \frac{\epsilon^{m n}}{e} \bar{\chi}_{m} \gamma_{5} \chi_{n}\right)
$$

The superspace transformations are constructed from the super-reparameterization and local Lorentz transformations of superspace. The infinitesimal changes in the vielbein and the connection under the superspace transformations are given by

$$
\begin{aligned}
& \delta E_{M}{ }^{A}=\xi^{N} \partial_{N} E_{M}{ }^{A}+\partial_{M} \xi^{N} E_{N}{ }^{A}+E_{M}{ }^{B} L_{B}{ }^{A} \\
& \delta \Omega_{M}=\xi^{N} \partial_{N} \Omega_{M}+\partial_{M} \xi^{N} \Omega_{N}-\partial_{M} L .
\end{aligned}
$$

From the superspace transformations of $E_{\mu}{ }^{A}$ and $\Omega_{\mu}$ one finds that the transformation parameters $\xi^{M}$ and $L$ can be decomposed as the following forms 16

$$
\xi^{m}=f^{m}-i \bar{\alpha} \gamma^{m} \theta+\frac{1}{4} \bar{\theta} \theta \bar{\alpha} \gamma^{n} \gamma^{m} \chi_{n}
$$




$$
\begin{aligned}
& \xi^{\mu}=\alpha^{\mu}-\frac{1}{2} \theta^{\nu}\left(\gamma_{5}\right)_{\nu}{ }^{\mu} l+\frac{i}{2} \bar{\alpha} \gamma^{m} \theta \chi_{m}{ }^{\mu}-\frac{1}{8} \bar{\theta} \theta \bar{\alpha} \gamma^{n} \gamma^{m} \chi_{n} \chi_{m}{ }^{\mu}+\frac{i}{4} \bar{\theta} \theta\left(\bar{\alpha} \gamma_{5} \gamma^{m}\right)^{\mu} \omega_{m}, \\
& L=l-\frac{i}{2} \bar{\alpha} \gamma_{5} \theta A-i \bar{\alpha} \gamma^{m} \theta \omega_{m}+\frac{1}{8} \bar{\theta} \theta \bar{\alpha} \gamma^{m} \gamma_{5} \chi_{m} A+\frac{i}{4} \bar{\theta} \theta \bar{\alpha} \gamma_{5} \psi+\frac{1}{4} \bar{\theta} \theta \bar{\alpha} \gamma^{n} \gamma^{m} \chi_{n} \omega_{m},
\end{aligned}
$$

where $(f, \alpha, l)$ correspond to coordinate, local supersymmetry and Lorentz transformations respectively. The supersymmetry transformations for the supergravity multiplet $\left(e_{m}{ }^{a}, \chi_{m}, A\right)$ may be read off and one finds

$$
\delta e_{m}{ }^{a}=i \bar{\alpha} \gamma^{a} \chi_{m}, \quad \delta \chi_{m}=2 \mathcal{D}_{m} \alpha+\frac{1}{2} \gamma_{m} \alpha A, \quad \delta A=\bar{\alpha} \psi
$$

where $\mathcal{D}_{m} \alpha=\partial_{m} \alpha-\frac{1}{2} \omega_{m} \gamma_{5} \alpha$ is the covariant derivative of the spinor $\alpha$.

In order to formulate the 2D SYM theory in superspace [17, 18, we need some constraints eliminating superflous components in the superfields $A_{B}(z)$, which are analogue of the supertorsion constraints (2.21) in the supergravity sector. According to the definite analogy between $2 \mathrm{D}$ and $4 \mathrm{D}$ gauge theory, one may choose the constraints as $F_{\alpha \beta}=0$. However we will find that the Bianchi identity (2.19) together with these constraints makes the 2D SYM theory trivial. In order to construct an appropriate nontrivial superspace, we instead impose the alternative constraints on the theory as follows:

$$
\left(\gamma_{a}\right)^{\alpha \beta} F_{\alpha \beta}=0
$$

Note that the above constraints can be solved by introducing the scalar superfield $W$

$$
F_{\alpha \beta}=\left(\gamma_{5}\right)_{\alpha \beta} W(z)
$$

Then the Eq.(2.18) implies that the scalar field strength $W(z)$ is represented in terms of the spinor potentials:

$$
W(z)=-\left(\gamma_{5}\right)^{\alpha \beta}\left(\mathcal{D}_{\alpha} A_{\beta}+i g_{Y M} A_{\alpha} A_{\beta}\right)
$$

Now one can similarly solve the Bianchi identity (2.19) or (2.20) subject to the constraints (2.29) and find that the Yang-Mills mutiplet can be expressed in terms of one spinor 
superfield $A_{\alpha}(z)$.2 The detailed solutions of the Yang-Mills Bianchi identity and the superspace formulation of 2D SYM theory coupled to supergravity will be presented to the Appendix B.

The spinor superfields $A_{\alpha}(z)$ correspond to the Yang-Mills vector multiplet in the adjoint representation of the gauge group $U(N)$ and are expanded as

$$
A_{\alpha}(z)=\eta_{\alpha}(\sigma)+i\left(\gamma^{m} \theta\right)_{\alpha} g_{m}(\sigma)+\theta_{\alpha} n(\sigma)+i\left(\gamma_{5} \theta\right)_{\alpha} \phi(\sigma)+\frac{1}{2} \bar{\theta} \theta b_{\alpha}(\sigma)
$$

where

$$
\begin{aligned}
& g_{m}(\sigma)=v_{m}-\frac{1}{2} \bar{\chi}_{m} \eta \\
& b_{\alpha}(\sigma)=\left(2 \lambda-\frac{1}{2} \gamma^{m} \gamma^{n} \chi_{m} g_{n}-i \gamma^{m} \Delta_{m} \eta+\frac{i}{2} \gamma^{m} \chi_{m} n-\frac{i}{4} A \eta+g_{Y M} \gamma_{5}[\phi, \eta]\right)_{\alpha}
\end{aligned}
$$

where $\Delta_{m} \eta=\partial_{m} \eta-\frac{1}{2} \omega_{m} \gamma_{5} \eta+i g_{Y M}\left[v_{m}, \eta\right]$. The gauge transformation on $A_{\alpha}(z)$, Eq.(2.14), is given by

$$
\begin{aligned}
\delta_{X} A_{\alpha}(z) & =E_{\alpha}{ }^{M}\left(\partial_{M} X+i g_{Y M}\left[A_{M}, X\right]\right), \\
& =E_{\alpha}{ }^{M} \nabla_{M} X
\end{aligned}
$$

where the scalar superfield $X(z)$ is a gauge parameter of $U(N)$ :

$$
X(z)=\omega(\sigma)+i \bar{\theta} \zeta(\sigma)+\frac{i}{2} \bar{\theta} \theta \rho(\sigma)
$$

As shown in the Appendix B, the Wess-Zumino (WZ) gauge [15], $\eta=n=0$, can be chosen by using the gauge freedoms $\zeta$ and $\rho$ and in this gauge the transformations (2.33) of the component fields $v_{m}, \phi$, and $\lambda$ reduce to the ordinary gauge transformations, so that they correspond to the Yang-Mills gauge field, adjoint scalar, and their superpartner, gaugino, respectively.

\footnotetext{
${ }^{2}$ In fact, it is not necessary to solve the Bianchi identities because, by solving the constraints (2.29) directly, we can easily determine the vector potential $A_{m}(z)$ in terms of spinor potential $A_{\alpha}(z)$.
} 
Next we consider the superspace transformation of the superfields $A_{\alpha}(z)$ defined as [15]

$$
\delta A_{\alpha}(z)=\xi^{M} \partial_{M} A_{\alpha}(z)-\frac{1}{2}\left(\gamma_{5}\right)_{\alpha}^{\beta} A_{\beta}(z) L(z)
$$

Since the above superspace transformation does not preserve the WZ gauge $\eta=n=0$, the gauge parameter $X(z)$ should be field-dependent and decomposed as the following form in order to preserve the WZ gauge

$$
X(z)=\omega(\sigma)+i \bar{\alpha} \gamma^{m} \theta v_{m}+i \bar{\alpha} \gamma_{5} \theta \phi+\frac{1}{2} \bar{\theta} \theta\left(\bar{\alpha} \lambda-\frac{1}{2} \bar{\alpha} \gamma^{m} \gamma^{n} \chi_{m} v_{n}+\frac{1}{4} \bar{\alpha} \gamma_{5} \gamma^{m} \chi_{m} \phi\right)
$$

In the WZ gauge the superfield $A_{\alpha}(z)$ has the following component expression:

$$
A_{\alpha}(z)=i\left(\gamma^{m} \theta\right)_{\alpha} v_{m}(\sigma)+i\left(\gamma_{5} \theta\right)_{\alpha} \phi(\sigma)+\bar{\theta} \theta\left(\lambda-\frac{1}{4} \gamma^{m} \gamma^{n} \chi_{m} v_{n}\right)_{\alpha}(\sigma)
$$

The supersymmetry transformation (2.34) for the Yang-Mills multiplet $\left(v_{m}, \phi, \lambda\right)$ becomes covariantized transformation in the WZ gauge and can be determined as (see Appendix B)

$$
\begin{aligned}
\delta v_{m}= & i \bar{\alpha} \gamma^{m} \lambda-\frac{i}{2} \bar{\alpha} \gamma_{5} \chi_{m} \phi-\frac{i}{4} \bar{\alpha} \gamma_{5} \gamma^{n} \gamma_{m} \chi_{n} \phi \\
\delta \phi= & i \bar{\alpha} \gamma_{5} \lambda-\frac{i}{4} \bar{\alpha} \gamma^{m} \chi_{m} \phi, \\
\delta \lambda= & \frac{1}{2} \gamma^{m} \gamma^{n} \alpha\left(v_{n m}+\frac{i}{2} \bar{\lambda} \gamma_{m} \chi_{n}-\frac{i}{2} \bar{\lambda} \gamma_{n} \chi_{m}\right)+\gamma_{5} \gamma^{m} \alpha\left(\nabla_{m} \phi+\frac{i}{2} \bar{\lambda} \gamma_{5} \chi_{m}\right) \\
& +\frac{1}{2} \gamma_{5} \gamma^{m}\left(\mathcal{D}_{m} \alpha-\frac{1}{4} \gamma_{m} \alpha A\right) \phi+\frac{i}{4} \bar{\alpha} \gamma_{5} \lambda \gamma_{5} \gamma^{m} \chi_{m} \\
& +\frac{i}{16} \bar{\chi}_{n} \gamma_{5} \gamma^{n} \gamma^{m} \gamma^{l} \chi_{m} \gamma_{l} \alpha \phi-\frac{i}{32} \bar{\chi}_{n} \gamma^{n} \gamma^{m} \chi_{m} \gamma_{5} \alpha \phi,
\end{aligned}
$$

where $v_{n m}=\partial_{n} v_{m}-\partial_{m} v_{n}+i g_{Y M}\left[v_{n}, v_{m}\right]$ is the Yang-Mills field strength.

\section{NON-ABELIAN RAMOND-NEVEU-SCHWARZ STRING THEORY}

According to the motivations mentioned in the Introduction, we will now try to construct consistent worldsheet formulation of RNS string theory where the "spacetime coordinates"

of strings are treated as non-commuting matrices. Our string coordinates $X^{I}(\sigma)$ are $N \times N$ 
hermitian matrices in the adjoint of an $U(N)$ group, carring also an $S O(D-1,1)$ vector index $I=0,1, \cdots, D-1.1$

In order to obtain a desirable formulation for the NARNS string, the adjoint matters in the gauge group $U(N)$, playing a role of matrix string coordinates, need to be introduced. Of course, this adjoint matter couples to the SYM field as well as the worldsheet supergraviton. We thus introduce worldsheet scalar superfields $V^{I}(z)$ in the adjoint representation of the $U(N)$ group, which have the expansion:

$$
V^{I}(z)=X^{I}(\sigma)+i \bar{\theta} \psi^{I}(\sigma)+\frac{i}{2} \bar{\theta} \theta F^{I}(\sigma)
$$

Note that the worldsheet spinor $\psi^{I}(\sigma)$ is an $S O(D-1,1)$ vector like as $X^{I}(\sigma)$. Under the superspace transformation, $V^{I}(z)$ changes as follows:

$$
\delta V^{I}(z)=\xi^{M} \partial_{M} V^{I}+i g_{Y M}\left[V^{I}, X\right]
$$

with the gauge parameter $X(z)$ given by Eq.(2.35). Inserting the explicit form (2.27) for $\xi^{M}$, one finds the supersymmetry transformation law for the component fields:

$$
\begin{aligned}
\delta X^{I} & =i \bar{\alpha} \psi^{I} \\
\delta \psi^{I} & =\gamma^{m} \alpha\left(\nabla_{m} X^{I}-\frac{i}{2} \bar{\chi}_{m} \psi^{I}\right)+\alpha F^{I}+i g_{Y M} \gamma_{5} \alpha\left[\phi, X^{I}\right] \\
\delta F^{I} & =i \bar{\alpha} \gamma^{m} \Delta_{m} \psi^{I}-\frac{i}{2} \bar{\alpha} \gamma^{n} \gamma^{m} \chi_{n}\left(\nabla_{m} X^{I}-\frac{i}{2} \bar{\chi}_{m} \psi^{I}\right)-\frac{i}{2} \bar{\alpha} \gamma^{m} \chi_{m} F^{I} \\
& \quad-g_{Y M} \bar{\alpha}\left[\lambda, X^{I}\right]-g_{Y M} \bar{\alpha} \gamma_{5}\left[\phi, \psi^{I}\right]-\frac{1}{4} g_{Y M} \bar{\alpha} \gamma_{5} \gamma^{m} \chi_{m}\left[\phi, X^{I}\right] .
\end{aligned}
$$

We endow our system with the natural metric which is invariant under $U(N) \times S O(D-$ $1,1)$ :

$$
|\delta X|^{2}=\int d^{2} \sigma g_{I J} \operatorname{Tr}\left(\delta X^{I} \delta X^{J}\right)
$$

where $g_{I J}$ is the Minkowski metric of the embedding space with signature (D-1,1).

\footnotetext{
${ }^{3}$ In next section we will give the reliable evidences that the critical dimension of the NARNS string is also 10 in a special limit.
} 
Under the metric (3.3), the gauge invariant locally supersymmetric worldsheet action is then given by

$$
I_{W S}=\frac{T}{2} \int d^{2} z E g_{I J} \operatorname{Tr}\left(E_{\alpha}{ }^{M} \nabla_{M} V^{I} E^{\alpha N} \nabla_{N} V^{J}\right)
$$

where $d^{2} z=d^{2} \sigma d \theta d \bar{\theta}$ is the superworldsheet volume and $\nabla_{M} V^{I}=\partial_{M} V^{I}+i g_{Y M}\left[A_{M}, V^{I}\right]$. The coupling constant $g_{Y M}$ should be proportional to the string tension as $\sqrt{T}=1 / \sqrt{2 \pi \alpha^{\prime}}$ for a dimensional reason. Thus we introduce the dimensionless coupling constant $g_{s}$ as

$$
g_{Y M}^{-2} \equiv g^{2}=\alpha^{\prime} g_{s}^{2}
$$

Then the weak coupling is characterized by the condition $g_{s}^{2}=2 \pi T g_{Y M}^{-2} \ll 1$.

In Sec.II, we observed that the spinor superfields $A_{\alpha}(z)$ may be viewed as the supersymmetric generalization of the Yang-Mills potentials. To construct the corresponding SYM theory, we need a gauge covariant supersymmetric field strength related with the usual Yang-Mills part. It is easily confirmed that this object is given by the covariant derivative of the superfield $W$ in the Eq.(B7). Hence we can easily write down the SYM action coupled to 2D supergravity:

$$
I_{Y M}=-\frac{1}{8} \int d^{2} z E \operatorname{Er}\left(E_{\alpha}{ }^{M} \nabla_{M} W E^{\alpha N} \nabla_{N} W\right)
$$

We must emphasize the fact that the "super Euler number" defined on a super Riemann surface $\mathcal{M}$ reduces to the standard Euler number

$$
\chi(\mathcal{M})=\frac{i}{2 \pi} \int_{\mathcal{M}} d^{2} z E S=\frac{1}{2 \pi} \int_{\mathcal{M}} d^{2} \sigma e R
$$

where $R=\frac{\epsilon^{m n}}{e} \partial_{m} \omega_{n}$ is the curvature of the connection $\omega_{m}$ in Eq.(2.24). This action contains no term involving the auxiliary field $A$ unlike the higher dimensional supergravity [15]. We will not consider the $2 \mathrm{D}$ pure supergravity action (3.6) since it is a total derivative and so have no dynamics.

From the Eq.(3.4) and Eq.(3.5), one can obtain the corresponding worldsheet action for the NARNS strings 


$$
\begin{aligned}
I=\int d^{2} \sigma e & \operatorname{Tr}\left\{\frac { T } { 2 } g _ { I J } \left(-g^{m n} \nabla_{m} X^{I} \nabla_{n} X^{J}-i \bar{\psi}^{I} \gamma^{m} \nabla_{m} \psi^{J}+i \bar{\chi}_{n} \gamma^{m} \gamma^{n} \psi^{I} \nabla_{m} X^{J}\right.\right. \\
& -\frac{1}{8} \bar{\chi}_{m} \gamma^{n} \gamma^{m} \chi_{n} \bar{\psi}^{I} \psi^{J}+F^{I} F^{J}+\frac{1}{2 g^{2}}\left[\phi, X^{I}\right]\left[\phi, X^{J}\right]+\frac{1}{g} \bar{\psi}^{I}\left[\lambda, X^{J}\right] \\
& \left.+\frac{1}{g} \bar{\psi}^{I} \gamma_{5}\left[\phi, \psi^{J}\right]+\frac{1}{4 g} \bar{\psi}^{I} \gamma_{5} \gamma^{m} \chi_{m}\left[\phi, X^{J}\right]\right)-\frac{g^{2}}{4} v_{m n} v^{m n}-\frac{i}{2} \bar{\lambda} \gamma^{m} \Delta_{m} \lambda \\
& -\frac{1}{2} g^{m n} \nabla_{m} \phi \nabla_{n} \phi+\frac{i}{4} g \bar{\lambda} \gamma_{5} \gamma^{m} \chi_{m} v-\frac{i}{4} \bar{\lambda} \gamma_{5} \chi^{m} \nabla_{m} \phi-\frac{3 i}{8} \bar{\lambda}_{5} \gamma^{n} \gamma^{m} \chi_{n} \nabla_{m} \phi \\
& -\frac{i}{8} \bar{\lambda} \gamma_{5} \gamma^{m} \gamma^{n} \mathcal{D}_{m} \chi_{n} \phi-\frac{i}{8} \bar{\chi}_{n} \gamma^{n} \gamma_{5} \gamma^{m} \Delta_{m} \lambda \phi-\frac{i}{8} \bar{\chi}_{p} \gamma^{p} \chi^{m} \phi \nabla_{m} \phi+\frac{g}{4} A v \phi \\
& +\frac{i}{32} \bar{\chi}_{p} \gamma^{p} \gamma^{m} \gamma^{n} \mathcal{D}_{m} \chi_{n} \phi \phi+\frac{3}{32} \bar{\chi}_{m} \gamma^{n} \gamma^{m} \chi_{n} \bar{\lambda} \lambda-\frac{i}{16} \bar{\chi}_{n} \gamma^{m} \gamma^{n} \chi_{m} A \phi \phi \\
& +\frac{3 i}{16} \bar{\lambda} \gamma_{5} \gamma^{m} \chi_{m} A \phi+\frac{i}{32} g \bar{\chi}_{n} \gamma^{n} \gamma^{m} \chi_{m} v \phi+\frac{1}{64} \bar{\chi}_{m} \gamma^{n} \gamma^{m} \chi_{n} \bar{\chi}^{p} \chi_{p} \phi \phi \\
& \left.+\frac{3}{64} \bar{\chi}_{m} \gamma^{n} \gamma^{m} \chi_{n} \bar{\lambda} \gamma_{5} \gamma^{p} \chi_{p} \phi+\frac{1}{8} A^{2} \phi \phi+\frac{1}{g} \bar{\lambda} \gamma_{5} \lambda \phi\right\}
\end{aligned}
$$

where $v=\frac{1}{e} \epsilon^{m n} v_{m n}$ and $\mathcal{D}_{m} \chi_{n} \equiv e_{n a} \mathcal{D}_{m} \chi^{a}=e_{n a}\left(\partial_{m} \chi^{a}-\frac{1}{2} \omega_{m} \gamma_{5} \chi^{a}+\omega_{m} \epsilon^{a b} \chi_{b}\right)$. The action (3.7) is automatically invariant under supersymmetry transformations, Eq.(2.28), Eq.(2.37), and Eq.(3.2) (up to total derivatives) because it was derived from a superspace formalism. The Yukawa type interaction, $g_{Y M} \bar{\lambda} \gamma_{5} \lambda \phi$, in Eq.(3.7) is the only term coming from the commutator in Eq.(3.5). The Yang-Mills coupling $g_{Y M}$ has been absorbed in the normalization of $v_{m}: v_{m} \rightarrow \frac{v_{m}}{g_{Y M}}$.

In two dimensions, the Yang-Mills gauge fields themselves have no propagating degrees of freedom - there are no gluons. This does not make the theory trivial, but the gauge field interactions give rise to a confining potential for colored objects [19]. In addition, the SYM multiplet in two dimensions contains genuine dynamical degrees of freedom in the adjoint representation. Thus 2D SYM theory may reveal nontrivial physical spectrums such as nonperturbative vacuum structures [18]. Although all this is true, the SYM action (3.5) is at most order of $\alpha^{\prime}$ compared to the worldsheet action (3.4). In infrared (IR) limit, i.e. $\alpha^{\prime} \rightarrow 0$ with fixed $g_{s}$, the SYM part can be thus ignored. It is important to observe that, in this limit, the SYM action (3.5) is strongly coupled and we expect a nontrivial CFT to describe the IR fixed point.

It turns out that we can find this CFT description via the following rather naive reasoning. We first notice that, in the $\alpha^{\prime} \rightarrow 0$ limit, the potential terms comming from the 
commutators in the NARNS string acton (3.7) effectively turn into constraints, requiring all the matrix fields in the adjoint representation of $U(N)$ to commute. This means that we can write the matrix coordinates $X^{I}$ in a simultaneously diagonalized form

$$
X^{I}=U^{-1} \operatorname{diag}\left(x_{1}^{I}, \cdots, x_{N}^{I}\right) U
$$

with $U \in U(N)$. Here $U$ and all eigenvalues $x_{a}^{I}$ can of course still depend on the worldsheet coordinates. In this IR limit our NARNS string theory has a free string limit where the usual RNS string theory is recovered and it becomes N-copies of usual RNS string [20]:

$$
\begin{gathered}
I_{R N S}=\frac{T}{2} \int d^{2} \sigma e g^{I J}\left(-g^{m n} \partial_{m} x_{I}^{a} \partial_{n} x_{J}^{a}-i \bar{\psi}_{I}^{a} \gamma^{m} \partial_{m} \psi_{J}^{a}+i \bar{\chi}_{n} \gamma^{m} \gamma^{n} \psi_{I}^{a} \partial_{m} x_{J}^{a}\right. \\
\left.-\frac{1}{8} \bar{\chi}_{m} \gamma^{n} \gamma^{m} \chi_{n} \bar{\psi}_{I}^{a} \psi_{J}^{a}+F_{I}^{a} F_{J}^{a}\right),
\end{gathered}
$$

where $a=1, \cdots, N$ is a Lie algebra index belonging only to Cartan subalgebra of $U(N)$. This action is invariant under general coordinate transformations, local Lorentz transformation, local supersymmetry transformations, Eq.(2.28) and Eq.(3.2) (with $g_{Y M}=0$ ), and Weyl transformation, under which the fields rescale as follows $(\Lambda=\Lambda(\sigma))$

$$
x_{I}^{a} \rightarrow x_{I}^{a}, \quad \psi_{I}^{a} \rightarrow \Lambda^{-1 / 2} \psi_{I}^{a}, \quad F_{I}^{a} \rightarrow \Lambda^{-1} F_{I}^{a}, \quad e_{m}^{b} \rightarrow \Lambda e_{m}^{b}, \quad \chi_{m} \rightarrow \Lambda^{1 / 2} \chi_{m} .
$$

Furthermore the action is invariant under the following transformation

$$
\chi_{m} \rightarrow \chi_{m}+\gamma_{m} \chi
$$

where $\chi(\sigma)$ is an arbitrary Majorana spinor. Using these eight gauge degrees of freedom, $e_{m}{ }^{a}$ and $\chi_{m}$ can be completely gauged away. In other words, we may choose the superconformal gauge, $e_{m}{ }^{a}=\delta_{m}{ }^{a}$ and $\chi_{m}=0$ [20]. Through the equation of motion of the auxiliary field $F_{I}^{a}$, we then arrive at the gauge fixed Polyakov action [21] whose quantum theory is given by superconformal field theory (SCFT)

\footnotetext{
${ }^{4}$ Although the constraint $\left[\phi, X^{I}\right]^{2}=0$ does not necessarily imply $\left[\phi, X^{I}\right]=0$ because of the $S O(D-1,1)$ metric $g_{I J}$, we will ignore the possibility.
} 


$$
I_{P}=\frac{T}{2} \int d^{2} \sigma g^{I J}\left(-\eta^{m n} \partial_{m} x_{I}^{a} \partial_{n} x_{J}^{a}-i \bar{\psi}_{I}^{a} \gamma^{m} \partial_{m} \psi_{J}^{a}\right)
$$

where $\eta^{m n}$ is a flat worldsheet metric.

It is interesting to observe that, in the $g_{s} \rightarrow 0$ limit with fixed $\alpha^{\prime}$, the SYM action (3.5) is invariant under a Weyl transformation that rescales the fields according to

$$
\phi \rightarrow \phi, \quad \lambda \rightarrow \Lambda^{-1 / 2} \lambda, \quad v_{m} \rightarrow v_{m}
$$

together with the super Weyl transformation of $E_{M}{ }^{A}$ which consists of the last two equations in Eq. (3.10) and $A \rightarrow \Lambda^{-1} A$ [16]. This fact can be most easily understood as following way: Since the terms involving with $v_{m n}$ vanish in the limit, the superfield $W$ in Eq.(3.5) exhibits the same behavior as the superfield $V^{I}$ in Eq.(3.4) under the conformal transformation (3.13). In addition, the Yukawa type interaction which breaks the conformal invariance vanishes in the limit because the gaugino $\lambda$ and the scalar $\phi$ should align in the direction of Cartan subalgebra of $U(N)$. Then the behavior of the SYM action under the super Weyl transformation is exactly the same as the action (3.4), which is superconformally invariant as proved by Howe [16]. This implies that the Weyl symmetry is preserved at least up to $\alpha^{\prime}$ order provided that the SYM multiplet scales as the Eq.(3.13). But the transformation (3.11) is no more symmetry of the action (3.5). It will be showed that this symmetry is recovered at a particular situation.

When the SYM action in the $g_{s} \rightarrow 0$ limit is considered, we can use the only six gauge degrees of freedom 5 and so may choose the gauge $e_{m}{ }^{a}=\delta_{m}{ }^{a}$ and $\chi_{m}=\gamma_{m} \chi$. In this gauge, the NARNS string action (3.7) becomes

$$
\begin{aligned}
I_{W e a k}=T \int d^{2} & \sigma \operatorname{Tr}\left\{\frac{1}{2} g^{I J}\left(-\eta^{m n} \nabla_{m} X_{I} \nabla_{n} X_{J}-i \bar{\psi}_{I} \gamma^{m} \nabla_{m} \psi_{J}\right)\right. \\
+ & 2 \pi \alpha^{\prime}\left(-\frac{1}{2} \eta^{m n} \nabla_{m} \phi \nabla_{n} \phi-\frac{i}{2} \bar{\lambda} \gamma^{m} \nabla_{m} \lambda\right. \\
& \left.\left.-\frac{i}{4} \bar{\chi} \gamma^{m} \partial_{m} \chi \phi \phi+\frac{3 i}{8} \bar{\lambda} \gamma_{5} \chi A \phi+\frac{1}{8} A^{2} \phi \phi\right)\right\} .
\end{aligned}
$$

\footnotetext{
${ }^{5}$ Although the NARNS string theory definitely has an additive $U(N)$ gauge symmetry, we would, for the present, keep the $U(N)$ symmetry.
} 
Note that, in the limit $g_{s} \rightarrow 0$, all the matrix fields in the action (3.14) except the YangMills gauge fields $v_{m}$ still take the diagonalized form such as $X^{I}$ in the Eq.(3.8). In order to remove the auxiliary field $A$, we use the equation of motion for $A$

$$
A=-\frac{3 i}{2} \bar{\lambda} \gamma_{5} \chi \phi / \phi \phi
$$

Then the action $I_{W e a k}$ reduces to the following form

$$
\begin{aligned}
I_{\text {Weak }}= & T \int d^{2} \sigma \operatorname{Tr}\left\{\frac{1}{2} g^{I J}\left(-\eta^{m n} \nabla_{m} X_{I} \nabla_{n} X_{J}-i \bar{\psi}_{I} \gamma^{m} \nabla_{m} \psi_{J}\right)\right. \\
& \left.+2 \pi \alpha^{\prime}\left(-\frac{1}{2} \eta^{m n} \nabla_{m} \phi \nabla_{n} \phi-\frac{i}{2} \bar{\lambda} \gamma^{m} \nabla_{m} \lambda\right)\right\} \\
+ & \frac{T}{4} \int d^{2} \sigma 2 \pi \alpha^{\prime} \operatorname{Tr}\left(-i \bar{\chi} \gamma^{m} \partial_{m} \chi \phi \phi+\frac{9}{16} \bar{\chi} \chi \overline{\tilde{\lambda}} \tilde{\lambda}\right),
\end{aligned}
$$

where $\tilde{\lambda}=\lambda^{a} \hat{\phi}^{a}, \quad \hat{\phi}^{a}=\phi^{a} / \sqrt{\phi \phi}$. As usual, we have the constraints comming from the equations of motion for the zweibein and gravitino due to the above gauge fixing:

$$
\begin{aligned}
T_{m n} & =\frac{1}{2} \operatorname{Tr}\left\{\nabla_{m} X^{I} \nabla_{n} X_{I}+i \bar{\psi}^{I} \gamma_{(m} \nabla_{n)} \psi_{I}+2 \pi \alpha^{\prime}\left(\nabla_{m} \phi \nabla_{n} \phi+i \bar{\lambda} \gamma_{(m} \nabla_{n)} \lambda+\frac{i}{8} \bar{\chi} \gamma_{(m} \partial_{n)} \chi \phi \phi\right)\right\} \\
& -\frac{1}{4} \eta_{m n} \operatorname{Tr}\left\{\nabla^{p} X^{I} \nabla_{p} X_{I}+i \bar{\psi}^{I} \gamma^{p} \nabla_{p} \psi_{I}+2 \pi \alpha^{\prime}\left(\nabla^{p} \phi \nabla_{p} \phi+i \bar{\lambda} \gamma^{p} \nabla_{p} \lambda\right)\right\}, \\
F_{m} & =\frac{i}{2} \operatorname{Tr}\left(\gamma^{n} \gamma_{m} \psi^{I} \nabla_{n} X_{I}\right)+\frac{1}{4} \pi \alpha^{\prime} \operatorname{Tr}\left(3 i \gamma_{5} \gamma^{n} \gamma_{m} \lambda \nabla_{n} \phi-2 i \partial_{m} \phi^{2}+i \gamma_{m} \gamma^{n} \partial_{n} \chi \phi^{2}+\frac{9}{8} \overline{\tilde{\lambda}} \tilde{\lambda} \chi\right) .
\end{aligned}
$$

The superconformal generators, $T_{m n}$ and $F_{m}$, no loger satisfy the tracelessness property

$$
\begin{aligned}
& T_{m}^{m}=\frac{i}{16} \bar{\chi} \gamma^{m} \partial_{m} \chi \phi \phi \\
& \gamma^{m} F_{m}=2 \pi \alpha^{\prime}\left(\frac{i}{4} \gamma^{m} \chi \partial_{m} \phi^{2}+\frac{i}{4} \gamma^{m} \partial_{m} \chi \phi^{2}+\frac{9}{64} \gamma^{m} \chi \overline{\tilde{\lambda}} \tilde{\lambda}\right)
\end{aligned}
$$

This is due to the fact the NARNS string action in the $g_{s} \rightarrow 0$ limit does not have the symmetry (3.11), thus full superconformal symmetry.

We would like to seek the particular situation for the superconformal symmetry of the NARNS string to be recovered. In order to satisfy this requirement, we must have the conditions, $T_{m}^{m}=\gamma^{m} F_{m}=0$. Interestingly, this can be achieved if only the following conditions are fufilled

$$
\tilde{\lambda}=\lambda^{a} \hat{\phi}^{a}=0, \quad \phi^{2}=\phi^{a} \phi^{a}=\text { constant }
$$


and together with the Dirac equation $\gamma^{m} \partial_{m} \chi=0$. In order for the constraint $\lambda^{a} \hat{\phi}^{a}=0$ not to completely break the worldsheet supersymmtry (2.37), we should have an another condition

$$
\bar{\lambda}^{a} \lambda^{a}+\frac{1}{4} \bar{\chi} \chi \phi^{a} \phi^{a}=0
$$

Together with the condition (3.18), this implies that the gaugino and the scalar field must parallely align in the $U(N)$ group space:

$$
\lambda^{a}= \pm \frac{1}{2} \chi \phi^{a}
$$

When the condition (3.19) is satisfied, we arrive at a configuration to recover the superconformal symmetry

$$
\lambda^{a}=\chi=0, \quad \phi^{2}=\phi^{a} \phi^{a}=\text { constant }
$$

Then the scalar field $\phi$ in the Yang-Mills multiplet becomes a singlet with respect to the worldsheet supersymmetry, i.e. $\delta \phi=0$, and behaves as a modular parameter of the theory.

Let us give some remarks for this interesting phenomenon. First, notice that the SYM gauge theory in two dimensions can be obtained by a dimensional reduction from the SYM gauge theory in three dimensions. The adjoint scalar field can be understood as the component of the gauge field in the compactified dimension. Note that the Yukawa interaction in the Eq.(3.7) is nothing but the gauge interaction in this compactified extra dimension. Second, from the Eq.(3.15), we observe that the scalar field $\phi$ in the Yang-Mills multiplet shows the same behavior as the string coordinates $X^{I}$, i.e., it behaves as if it is an another coordinate of the string. If we would interpret the scalar field as the field living on a some compactified dimension, we can introduce a new coordinate along this compactified direction as

$$
X_{D+1}=\sqrt{2 \pi \alpha^{\prime}} \phi
$$

If the Abelianized string theory (3.9) is defined in the ten dimensions, the coordinate $X_{D+1}$ in the Eq.(3.21) should be involved with the eleven dimensional coordinate. Then the 
condition (3.20) constrains that the eleven dimensional coordinate $X_{D+1}$ should be defined on an (N-1)-dimensional orbifold $S^{N-1} / S_{N}$, where the orbifold group $S_{N}$ comes from the discrete Weyl symmetry of $U(N)$. It is prudently expected that our NARNS string theory has the natural M-theory interpretation and is related with the Matrix string theory.

\section{NARNS STRING AS MATRIX STRING THEORY}

In the previous section we showed that our NARNS string theory in the $\alpha^{\prime} \rightarrow 0$ limit with fixed $g_{s}$ has a free string limit where the usual RNS string theory is recovered and it becomes N-copies of usual RNS string. And we observed that, in the weak coupling limit, i.e. $g_{s} \rightarrow 0$, a new additional dimension appears in the string spectrum and it can be speculatively interpreted as the compactified eleven dimensional coordinate. In this section, we will argue that the NARNS string theory in the $\alpha^{\prime} \rightarrow 0$ limit with fixed $g_{s}$ can be described by the orbifold conformal field theory, which seems to correspond to the manifestly covariant worldsheet version of the MST of Dijkgraaf, Verlinde and Verlinde (DVV) [8]. And we observe that, in the weak coupling limit, i.e. $g_{s} \rightarrow 0$, the dynamics of a new additional eleven dimensional coordinate is given by an $S_{N^{-}}$orbifold $\mathrm{O}(\mathrm{N})$ sigma model. Our speculation in this section are preliminary and conjectural. We hope our conjectural speculations to be completed by the detailed analysis in the near future.

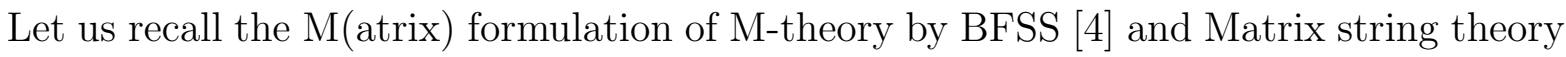
by DVV [8]. Consider an M-theory excitation on $\mathbf{R}^{10} \times S^{1}$ with finite mass $m$ which satisfies a Lorentz invariant eleven dimensional dispersion relation

$$
-P^{M} P_{M}=E^{2}-P_{11}^{2}-\vec{P}_{\perp}^{2}=m^{2}
$$

where the eleven dimensional momentum $P_{11}$ along the circle $S^{1}$ with radius $R_{11}$ should be quantized as $P_{11}=N / R_{11}$. In an IMF boosted along the $S^{1}, P_{11} \rightarrow \infty$, and a particular decompactified limit, i.e. $R_{11} \rightarrow \infty$, the dispersion relation (4.1) with the eleven dimensional Lorentz invariance effectively reduces to the transverse 9-dimensional Galilean dynamics: 


$$
H_{\perp}=E-P_{11}=\frac{\vec{P}_{\perp}^{2}}{2 P_{11}} .
$$

This relation implies that M-theory quantum dynamics in IMF may be captured by the quantum mechanics of particles with the mass $m=P_{11}$. What is the M-theory object on $\mathbf{R}^{10} \times S^{1}$ carrying the mass $P_{11}$ ? According to Witten [2], we see that this is just a D0brane, Kaluza-Klein excitation along the eleven dimensional circle $S^{1}$, whose IR dynamics is given by the super-Yang-Mills quantum mechanics 22] defined in 9 space dimensions $\mathbf{R}^{9}\left(X^{i}, i=1, \cdots, 9\right)$. Thus the following conjecture can be made:

M-theory quantum dynamics in IMF is described by the D0-brane quantum mechanics.

This is exactly the BFSS conjecture 4] which almost has passed many nontrivial tests so far.

What Witten has shown is that the strong coupling limit of type IIA superstring theory should be identical to the M-theory on $\mathbf{R}^{10} \times S^{1}$ [2]. According to this ficture, if we consider the BFSS matrix theory more compactified on a circle $S^{1}$ of radius $R_{9}$ along the $X^{9}$ direction, and if we think of dimension 9 rather than dimension 11 as the M-theory compactification direction to get the type IIA theory, the SYM theory in two dimensions should provide a light-front description of the type IIA string theory according to the duality relation [23]

$$
I I A \sim M \text { on } S^{1} \sim M(\text { atrix }) \text { on } \tilde{S}^{1}
$$

where the radius $\tilde{R}_{9}$ of the dual circle $\tilde{S}^{1}$ is related by $\tilde{R}_{9}=1 / 2 \pi R_{9}$. Since the dimension 9 is the M-theory compactification direction, the fumdamental objects which carry the lightfront momentum $p^{+}=N / R_{11}$ are no longer D0-branes, but rather strings. Thus this gives the DVV description on MST [8], namely that 2D Yang-Mills theory in the large N limit should correspond to light-front type IIA string theory which is given by a sigma model on the orbifold target space $\left(\mathbf{R}^{8}\right)^{N} / S_{N}$ as $R_{9} \rightarrow 0$. The Weyl symmetry $S_{N}$ is the discrete remnant of the gauge group $U(N)$ acting within the Cartan subalgebra, indicating that the string bits, partons carrying a minimum unit of light-front momemtum, should be treated 
as indistinguishable objects. One particularly nice picture of MST comes in following way. Since the string configuration may respect the residual gauge symmetry $S_{N}$ if we go around the space-like $S^{1}$ of the worldsheet, the matrix configuration need not be periodic in $\sigma$. The matrices $X^{i}(0)$ and $X^{i}(2 \pi)$ can be related by an arbitrary permutation. The lengths of the cycles of this permutation determine the numbers of string bits, which combine into long strings whose longitudinal momentum $p^{+}=n / R_{11}$ can become large in the large $\mathrm{N}$ limit. The twisted sectors of this theory correspond precisely to the sectors where the string bits are combined in different permutations.

We want to construct a theory with "space-time" Poincaré symmetry as well as $U(N)$ gauge symmetry. The Poincaré symmetry for the matrix coordinates $\left\{X_{I}\right\}$ means the global $S O(D-1,1)$ symmetry plus the translations acting on the matrices $X_{I}$ by $X_{I} \rightarrow X_{I}+$ $a_{I} \cdot I_{N \times N}$. The NARNS string action (3.7) manifestly has this global "space-time" Poincaré symmetry. The motion of the center of mass of the system associated with the global shift $X_{I} \rightarrow X_{I}+\operatorname{Tr} X_{I} / N \cdot I_{N \times N}$ comes from the $U(1)$ part by separating off the trace part of $U(N)$, i.e. $U(N)=U(1) \times S U(N)$. Thus $S U(N)$ matrices describe the relative motion of the system [4].

In this section we take string unit $\alpha^{\prime}=2$ and the two dimensional worldsheet is taken to be a cylinder parameterized by the coordinates $(\tau, \sigma)$ with $\sigma$ between 0 and $2 \pi$. The light-cone matrix coordinates are defined to be $X^{ \pm}=\frac{1}{\sqrt{2}}\left(X^{0} \pm X^{D-1}\right), \psi^{ \pm}=\frac{1}{\sqrt{2}}\left(\psi^{0} \pm \psi^{D-1}\right)$ and $X^{i}, \psi^{i}, i=1, \cdots, D-2$ and the scalar product in terms of light-cone components is $V \cdot W=-V^{+} W^{-}-V^{-} W^{+}+V^{i} W^{i}$.

In IR limit, i.e. $\alpha^{\prime} \rightarrow 0$ with fixed $g_{s}$, , where $g_{Y M} \rightarrow \infty$, since the 2D Yang-Mills theory is also a confining phase [19], only observable entries, that can escape the confining potential, are diagonalized matrix fields. Thus the usual spacetime picture emerges when

\footnotetext{
${ }^{6}$ We have taken the string unit $\alpha^{\prime}=2$. Here the $\alpha^{\prime} \rightarrow 0$ limit means that the typical length scale under consideration is very very large compared to the string scale $\alpha^{\prime}=2$.
} 
all of the $X_{I}$ commute with each other, hence can be simultaneously diagonalized as the indistinguishable $N$-tuple points $X^{I}=\operatorname{diag}\left(x_{a}^{I}\right)$. In this limit, the SYM part disappears in the string spectrum and the $U(N)$ gauge symmetry is generically broken down to $U(1)^{N}$ with the Weyl group, the residual discrete symmetry of the gauge group, acting on the eigenvalues in Eq.(3.8). Therefore the light-cone SCFT of the Polyakov action (3.12) is given by the N-tuple transverse fields $x_{i}^{a}, \psi_{i}^{a}, i=1, \cdots, D-2, a=1, \cdots, N$ which define the orbifold target space given by the symmetric product space

$$
S^{N} \mathbf{R}^{D-2}=\left(\mathbf{R}^{D-2}\right)^{N} / S_{N}
$$

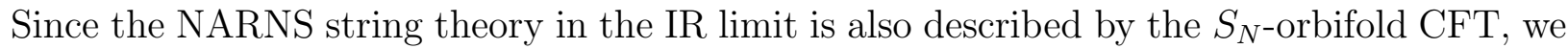
can follow the exactly same route taken by [24] and [8], keeping in mind some issues such as the GSO projection [25] and the level-matching condition [26] discussed later. In the Ref. [24], it is shown that this orbifold conformal field theory exactly corresponds to a second quantized string theory on the space $\mathbf{R}^{D-2} \times S^{1}$. Based on the exact equivalence between a second-quantized string spectrum and the spectrum of a $2 \mathrm{D} S_{N^{-}}$orbifold sigma model, this correspondence is more elaborated in [8] as Matrix string theory described above. In this correspondence, the Hilbert space of the orbifold conformal field theory is decomposed into twisted sectors $\mathcal{H}_{g}$ labeled by the conjugacy classes $[g]$ of the orbifold group $S_{N}$. The conjugacy classes $[g]$ are characterized by partitions $\left\{N_{n}\right\}$ of $\mathrm{N}$

$$
\sum_{n} n N_{n}=N
$$

where $N_{n}$ denotes the multiplicity of the cyclic permutation $(n)$ of $n$ elements in the decomposition of $g$

$$
[g]=(1)^{N_{1}}(2)^{N_{2}} \cdots(s)^{N_{s}}
$$

In each twisted sector, one must further keep only the states invariant under the centralizer subgroup $C_{g}$ of $g$, which takes the form

$$
C_{g}=\prod_{n=1}^{s} S_{N_{n}} \times \mathbf{Z}_{n}^{N_{n}}
$$


Here each factor $S_{N_{n}}$ permutes the $N_{n}$ cycles $(n)$, while each $\mathbf{Z}_{n}$ acts within one particular cycle $(n)$. Thus the total orbifold Hilbert space takes the form

$$
\mathcal{H}\left(S^{N} \mathbf{R}^{D-2}\right)=\bigoplus_{[g]} \mathcal{H}_{g}^{C_{g}},
$$

where $C_{g}$ invariant subspace $\mathcal{H}_{g}^{C_{g}}$ can be decomposed into the product over the subfactors $(n)$ of $N_{n}$-fold symmetric tensor products of appropriate smaller Hilbert spaces $\mathcal{H}_{(n)}^{\mathbf{Z}_{n}}$

$$
\mathcal{H}_{g}^{C_{g}}=\bigotimes_{n>0} S^{N_{n}} \mathcal{H}_{(n)}^{Z_{n}}
$$

The Hilbert spaces $\mathcal{H}_{(n)}^{\mathbf{Z}_{n}}$ in (4.9) denote the $\mathbf{Z}_{n}$ invariant subspace of a single string on $\mathbf{R}^{D-2} \times S^{1}$ with winding number $n$. We can represent this space using $n$ coordinate fields $x_{a}(\sigma) \in \mathbf{R}^{D-2}$ with the cyclic boundary condition

$$
x_{a}(\sigma+2 \pi)=x_{a+1}(\sigma)
$$

for $a \in(1, \cdots, n)$. We can glue the $n$ coordinate fields $x(\sigma)$ together into one single string field $x(\sigma)$ defined on the interval $0 \leq \sigma \leq 2 \pi n$. Hence, the oscillators of the long string that generate $\mathcal{H}_{(n)}^{\mathbf{Z}_{n}}$ have a fractional $1 / n$ moding relative to the string with winding number one. The group $\mathbf{Z}_{n}$ is generated by the cyclic permutation

$$
\omega: x_{a} \rightarrow x_{a+1}
$$

which via (4.10) corresponds to a translation $\sigma \rightarrow \sigma+2 \pi$. Thus the $\mathbf{Z}_{n}$-invariant subspace consists of those states for which the fractional left-moving minus right-moving oscillator numbers combined add up to an integer.

In the $S_{N}$-orbifold CFT, the twisted sectors of the orbifold corresponding to the possible multistring states are all superselection sectors unless string interactions are introduced that generate the elementary joining and splitting of strings. Thus the GSO projection summing over all possible spin structures for the string amplitudes [26] independently applies to each string in each superselection sector. This projection is performed separately on left and right movers because the states of the closed NARNS superstring are direct products of the 
Fock space states for the right and left movers. This degree of freedom gives us two types of string theory, i.e. type IIA and type IIB string theories. Then our NARNS string theory impartially should provide the matrix formulation of both type IIA and type IIB string theories. Since each string with lengths $n$ is the same as the usual RNS strings with same lengths, this reasoning then leads to the important conclusion that the critical dimension of the NARNS string theory in the IR limit is also 10 in which case superconformal symmetry is manifest even in the quantum level [21]. So we will restrict to the case $\mathrm{D}=10$.

Let us compactify the light-like coordinate $X^{-}=\frac{1}{\sqrt{2}}\left(X^{0}-X^{D-1}\right)$ on a circle of radius $\mathrm{R}$, which is essentially taken to infinity in order to obtain a uncompactified limit. In this case the conjugate momentum $p^{+}$is quantized as $p^{+}=N^{\prime} / R$ with $N^{\prime}$ being integer valued. This step introduces the twisted sectors corresponding to strings wound around the peoriodically identified coordinate $X^{-}$, each describing a set of noninteracting strings of length proportional to the carrying light-front momentum $n / R$ satisfying $N_{1}+2 N_{2} \cdots+n N_{n}=N^{\prime}$. For an instructive discussion on discrete light-cone quantization of string theory, see [27]. Until now, it seems that there is no relation between the light-front momentum $N^{\prime}$ and the dimension of gauge group $N$. But, according to the string bit or parton picture, considering the fact that the lengths $n$ of the individual strings specifies its light-cone momentum and the string Fock space is characterized by an integer $N^{\prime}$ satisfying $\sum_{n} n N_{n}=N^{\prime}$, it is resonable to identify $N$ with $N^{\prime}$. The invariance under $\mathbf{Z}_{n}$ implies that $N_{L}^{(n)}-N_{R}^{(n)}$ is a multiple of $n$ where $N_{L}^{(n)}, N_{R}^{(n)}$ are the usual oscillator level numbers of the strings with lengths $n$ [8]. In the limit $N \rightarrow \infty$, we obtain the usual level matching conditions $N_{L}^{(n)}-N_{R}^{(n)}=0$ since all the string states for which $N_{L}^{(n)}-N_{R}^{(n)} \neq 0$ becomes infinitely massive at large $\mathrm{N}$ and $\alpha^{\prime} \rightarrow 0$ limit.

Now we will consider an another limit, $g_{s} \rightarrow 0$ limit with fixed $\alpha^{\prime}$, where the SYM part cannot be ignored. In the previous section, we showed that the configuration (3.20) preserves the full superconformal symmetry, so the superconformal gauge fixing can be made: $e_{m}{ }^{a}=\delta_{m}{ }^{a}$ and $\chi_{m}=0$. In addition we will fix the $U(N)$ gauge symmetry as $v_{m}=0$. In this gauge the action (3.15) takes the CFT limit 


$$
I_{C F T}=-\frac{1}{8 \pi} \int d^{2} \sigma \operatorname{Tr}\left\{\partial^{m} X^{I} \partial_{m} X_{I}+i \bar{\psi}^{I} \gamma^{m} \partial_{m} \psi_{I}+\frac{4 \pi}{\lambda_{\phi}^{2}} \partial^{m} \hat{\phi} \partial_{m} \hat{\phi}\right\}
$$

with the generators

$$
\begin{aligned}
T_{m n}= & T_{m n}^{O C F T}+T_{m n}^{S^{N-1}} \\
= & \frac{1}{2} \operatorname{Tr}\left\{\partial_{m} X^{I} \partial_{n} X_{I}+i \bar{\psi}^{I} \gamma_{(m} \partial_{n)} \psi_{I}-\frac{1}{2} \eta_{m n}\left(\partial^{p} X^{I} \partial_{p} X_{I}+i \bar{\psi}^{I} \gamma^{p} \partial_{p} \psi_{I}\right)\right. \\
& \left.+\frac{4 \pi}{\lambda_{\phi}^{2}}\left(\partial_{m} \hat{\phi} \partial_{n} \hat{\phi}-\eta_{m n} \frac{1}{2} \partial^{p} \hat{\phi} \partial_{p} \hat{\phi}\right)\right\}, \\
F_{m}= & \frac{i}{2} \operatorname{Tr}\left(\gamma^{n} \gamma_{m} \psi^{I} \partial_{n} X_{I}\right),
\end{aligned}
$$

where we have identified the coupling constant $\lambda_{\phi}=1 / \sqrt{\phi \phi}$ with the inverse radius of the sphere $S^{N-1}$. Note that the gauge currents $J_{m}^{a} \equiv \delta I_{W e a k} / \delta v_{a}^{m}$ identically vanish because the $U(N)$ guage symmetry was generically broken down to $U(1)^{N}$ in the limit under consideration. Note that we have still discrete Weyl symmetry $S_{N}$ acting on the eigenvalues of matrix fields. After going to light-cone gauge $X^{+}=2 p^{+} \tau$ (where $p^{+}$is a diagonalized $\mathrm{N} \times$ $\mathrm{N}$ matrix), $\psi^{+}=0$ which fixes the gauge completely [26], we can solve the constraints (4.13) to determine the coordinates $X^{-}$and $\psi^{-}$as

$$
\begin{aligned}
& \delta_{m, 0} \partial_{n} X^{-}+\delta_{n, 0} \partial_{m} X^{-}+\eta_{n m} \partial_{0} X^{-} \\
& =\frac{1}{2} p^{+-1} \operatorname{Tr}\left\{\partial_{m} X^{i} \partial_{n} X^{i}+i \bar{\psi}^{i} \gamma_{(m} \partial_{n)} \psi^{i}-\frac{1}{2} \eta_{m n}\left(\partial^{p} X^{i} \partial_{p} X^{i}+i \bar{\psi}^{i} \gamma^{p} \partial_{p} \psi^{i}\right)\right. \\
& \left.\quad+\frac{4 \pi}{\lambda_{\phi}^{2}}\left(\partial_{m} \hat{\phi} \partial_{n} \hat{\phi}-\eta_{m n} \frac{1}{2} \partial^{p} \hat{\phi} \partial_{p} \hat{\phi}\right)\right\}, \\
& \psi^{-}=\frac{1}{2} p^{+-1} \operatorname{Tr}\left(\gamma^{n} \gamma^{0} \psi^{i} \partial_{n} X^{i}\right),
\end{aligned}
$$

which leaves only the transverse components $X^{i}, \psi^{i}$ and $\hat{\phi}$ as independent degrees of freedom (assuming that $\hat{\phi} \in S^{N-1} / S_{N}$ ). In terms of these transversal degrees of freedom only, the light-cone action is simply

$$
I_{L C}=-\frac{1}{8 \pi} \int d^{2} \sigma \operatorname{Tr}\left\{\partial^{m} X^{i} \partial_{m} X^{i}+i \bar{\psi}^{i} \gamma^{m} \partial_{m} \psi^{i}+\frac{4 \pi}{\lambda_{\phi}^{2}} \partial^{m} \hat{\phi} \partial_{m} \hat{\phi}\right\}
$$

The light-cone action (4.15) consists of two parts. The $\mathrm{N} \times \mathrm{N}$ matrix fields $X^{i}$ and $\psi^{i}$ transform in the $\boldsymbol{8}_{v}$ representation of the transversal $\mathrm{SO}(8)$ symmetry group. Apart from these 
fields, we have the scalar field $\hat{\phi}$ defined on the orbifold $S^{N-1} / S_{N}$ which can be identified with the coset space $S^{N-1} / S_{N} \cong S O(N) / S O(N-1) \times S_{N}$ as the target manifold. The diagonalized matrix fields $X^{i}$ and $\psi^{i}$ define the $S_{N}$-orbifold CFT on the N-fold symmetric product space $S^{N} \mathbf{R}^{8}=\left(\mathbf{R}^{8}\right)^{N} / S_{N}$ which has been already discussed.

The scalar fields $\hat{\phi}$ define so-called $S_{N}$-orbifold $\mathrm{O}(\mathrm{N})$ sigma model. The most simplest nontrivial example is a $\mathbf{Z}_{2}$-orbifold $O(2)$ sigma model which has been extensively studied so far [32,33]. This model describes a free massless scalar field $\hat{\phi}$ compactified on the orbifold $S^{1} / \mathbf{Z}_{2}$, known to describe the critical line of the Ashkin-Teller model, i.e. two Ising models coupled by a four spin interaction. Recall that the circle $S^{1}$ is just the quotient of an infinite line $\mathbf{R}$ by a discrete group $\Gamma=2 \pi R \mathbf{Z}$, i.e. $S^{1} \cong \mathbf{R} / \Gamma$. This orbifold $\mathrm{O}(2)$ model exhibits a quite interesting property, say that the $R=\sqrt{2}$ torus model, compactified on a circle instead of an orbifold, which has an $S U(2) \times S U(2)$ symmetry and the $R=1$ orbifold model, which is equivalent to two decoupled Ising models and consequently carries a representation of two $c=\frac{1}{2}$ Virasoro algebras, is exactly equivalent to each other [32,33]. It is a kind of an electric-magnetic S-duality relating the models at $R$ and $2 / R$, while, in our case, it is a T-duality [12].

If a Wess-Zumino-Witten (WZW) term is included with the kinetic energy term, this theory can be described by the gauged WZW theory based on the coset model $S O(N) / S O(N-1) \times S_{N}$ using the Goddard, Kent, and Olive algebraic construction [28]. This model provides a interesting property that the purely bosonic model is equivalent to a free fermion theory at a particular infrared fixed point [29]. It has been known by the explicit analysis of $\mathrm{O}(4)$ model with no orbifold group [30] that the radius of $S^{3}$ increases with energy, and as a result the local curvature tends to zero and in the infrared limit the radius decreases with decreasing energy, but it stops decreasing as it reaches its minimum critical radius which is the infrared fixed point having the fermionic description. Although the presence of these interesting phenomena, we don't completely identify the spectrum of this theory with the string theory aspects, particularly involving with the mysterious eleven 
dimensional M-theory.

\section{CONCLUSIONS}

According to the recent remarkable picture, so-called noncommutative spacetime geometry, appeared in nonperturbative string theory and M(atrix)-theory, we, in this paper, considered NARNS superstring theory as a generalization of usual RNS string theory. It is a 2D supergravity theory coupled to SYM fields and adjoint matters in a gauge group $U(N)$. Therefore the string coordinates of our theory are noncommuting matrices in the group $U(N)$. In a region that the usual spacetime picture emerges, this theory is described by the orbifold conformal field theory, essentially second quantized string theory in large $\mathrm{N}$ limit, contrary to the ordinary RNS string theory which has a first quantized description.

In the weak coupling limit that the SYM part must be considered and the superconformal symmetry is preserved, a new additional dimension appeared in the string spectrum, which is interpreted as compactified eleven dimension in this paper. This additional degree of

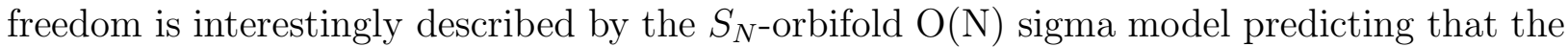
size of this dimension increases in the ultraviolet limit and decreases in the infrared limit 14. If a topological WZW term is included with the kinetic energy term, the size of the eleven dimension flows to some critical value in the IR limit instead of flowing to zero size, where the purely bosonic model is equivalent to a free fermion theory. But we don't ensure how the WZW term can be naturally introduced in the NARNS string action. It will be interesting for these phenomena to give a natural M-theory interpretation.

Our matrix model is a worldsheet formulation compared to the Green-Schwarz formulation of MST by DVV. While the MST of DVV is the type IIA string theory in the GreenSchwarz light-cone formulation, it seems that the NARNS string theory provides a (non)perturbative worldsheet matrix formulation of both type IIA and IIB superstring theory. The distinction of the type IIA and type IIB comes from the GSO projection independently performed on the right and left movers. The interaction of the NARNS string will be repre- 
sented by a local operator via a perturbation of the $S_{N}$-orbifold conformal field theory [8]. Thus the identification of the vertex operator generating this interaction would be important to obtain a picture of interacting NARNS string. According to the recent argument [31], the interaction generating the elementary joining and splitting of strings may come from the holonomy of the gauge fields $v_{m}$ through the monodromy of a twisted bundle. This implies that the gauge field on the string worldsheet is crucial in the dynamics of the theory.

Our theory also has two parameters like as MST [8], $\alpha^{\prime}$ and $g_{s}$, related with the string tension $T=1 / 2 \pi \alpha^{\prime}$ and the Yang-Mills coupling constant $g_{Y M}^{-2}=\alpha^{\prime} g_{s}^{2}$. The coupling constant $g_{s}$ precisely plays the same role with the string coupling constant $\lambda_{s}=e^{-(\text {vev of dilaton) }}$. If the string interactions generating the elementary joining and splitting of strings are also correctly reproduced in our model, the coupling constant $g_{s}$ can be directly related to the string coupling $\lambda_{s}$, since, in going from the one-string to the two-string and vice versa, the genus of the string worldsheet (Riemann surfaces) changes by one unit, so the vertex operator generating this interaction in our model must contain the factor $\lambda_{s}=e^{- \text {(vev of dilaton) }}$.

Compactification of the NARNS string theory means that some "matrix" string coordinates are defined over some compactified manifold. For example, if we compactify a coordinate $X^{I}$ on a circle $S^{1}$ of radius $R$, the components $X_{i j}^{I}$ (where the matrix indices $i$ and $j$ run from 1 to $\mathrm{N}$ ) of the matrix $X^{I}$ can then be expanded in terms of the Fourier modes over $S^{1}$

$$
X_{i j}^{I}(x)=\sum_{n} X_{i j, n}^{I} e^{i n x / \hat{R}}, \quad x \in S^{1}, n \in \mathbf{Z}
$$

where $\hat{R}=1 / 2 \pi R$. The $n$-th Fourier mode $X_{i j, n}^{I}$ exactly corresponds to the matrix component $X_{0 i, n j}^{I}$ of Ref. 23] which are based on the D0-brane basis where $i$ and $j$ are labels of N D0-branes and the coordinates $X_{m i, n j}^{I}$ are open string coordinates connecting the $i$-th D0-brane within block $m \in \mathbf{Z}$ and $j$-th D0-brane within $n$-th block. Therefore the compactification scenario of [23] can be equally applied to the NARNS string theory.

Although our matrix formulation of string theory is restricted only to the case of closed strings with $(1,1)$ worldsheet supersymmetry, e.g. type IIA and type IIB, it seems to be 
possible to extend to the cases of heterotic and type I strings. A naive conjecture for heterotic string and type I string is following: The matrix fields of the heterotic string are only 10dimensional coordinates, $X_{R, L}^{0, \cdots, 9}$ and $\psi_{R}^{0, \cdots, 9}$ and the compactified left-moving coordinates $X_{L}^{1, \cdots, 16}$ still remain C-number fields since they must identically generate the usual anomaly free gauge group $S O(32)$ and $E_{8} \times E_{8}$ for the consitituent strings. And, for the type I string, the identical $S O(32)$ Chan-Paton factor is globally assigned for the consitituent strings at the "boundary" of the strings and $\mathbf{Z}_{2}$ modding of type IIB matrix string theory. However it is not obvious how this naive scenario consistently works.

\section{ACKNOWLEDGEMENTS}

This work was supported by the Korean Science and Engineering Foundation through Center for Theoretical Physics, by the Korean Ministry of Education (BSRI-97-2414), and also by the Sogang University Research Grant in 1997.

\section{APPENDIX A: CONVENTIONS AND IDENTITIES}

The 2D bosonic metric is $\eta_{a b}$

$$
\eta^{a b}=\operatorname{diag}(-1,+1) \text { and } \epsilon^{a b}=-\epsilon^{b a} ; \epsilon^{01}=1
$$

The fermionic metric is $\epsilon^{\alpha \beta}$

$$
\epsilon^{\alpha \beta}=\epsilon_{\alpha \beta}=-\epsilon^{\beta \alpha} ; \epsilon^{12}=1 \text {. }
$$

The spinors in this paper are Majorana in any case. Spin indices are raised and lowered by $\epsilon^{\alpha \beta}$ according to the rules

$$
\theta^{\alpha}=\epsilon^{\alpha \beta} \theta_{\beta}, \quad \psi_{\alpha}=\psi^{\beta} \epsilon_{\beta \alpha}, \quad \bar{\theta} \psi \equiv \theta^{\alpha} \psi_{\alpha}
$$

The Dirac $\gamma$-matrices are represented in the Majorana and Weyl basis: 


$$
\begin{aligned}
& \gamma^{a} \gamma^{b}+\gamma^{b} \gamma^{a}=2 \eta^{a b}, \\
& \gamma^{0}=\left(\begin{array}{cc}
0 & 1 \\
-1 & 0
\end{array}\right), \quad \gamma^{1}=\left(\begin{array}{ll}
0 & 1 \\
1 & 0
\end{array}\right), \quad \gamma_{5}=\gamma^{0} \gamma^{1}=\left(\begin{array}{cc}
1 & 0 \\
0 & -1
\end{array}\right)
\end{aligned}
$$

The index structure of the Dirac matrices is $\left(\gamma^{a}\right)_{\alpha}^{\beta}$. The bilinear form $\bar{\psi}_{1} \Gamma \psi_{2}$ for spinors $\bar{\psi}_{1}$ and $\psi_{2}$ where $\Gamma$ is any combination of $\gamma$-matrices means that

$$
\bar{\psi}_{1} \Gamma \psi_{2}=\psi_{1}^{\alpha}(\Gamma)_{\alpha}^{\beta} \psi_{2 \beta}
$$

The D-dimensional target space metric is $g^{I J}$

$$
g^{I J}=\operatorname{diag}(-1,+1, \cdots,+1) .
$$

The Cartan-Killing metric for $U(N)$ Lie group in Eq.(2.13) and its Lie algebra are following

$$
\operatorname{Tr}\left(T^{r} T^{s}\right)=\delta^{r s}, \quad\left[T^{r}, T^{s}\right]=i f^{r s p} T^{p}
$$

where $f^{r s p}$ is the structure constant of semi-simple Lie algebra $s u(N)$.

Finally, we list the useful identies

$$
\begin{aligned}
& \left(\bar{\psi}_{1} \psi_{2}\right) \psi_{3}=-\frac{1}{2} \sum_{A}\left(\bar{\psi}_{1} \Gamma^{A} \psi_{3}\right) \Gamma_{A} \psi_{2}, \text { where } \Gamma^{A}=\left(\mathbf{1}, \gamma^{a}, \gamma_{5}\right) \\
& \gamma^{a} \gamma^{b} \gamma_{a}=0 \\
& \bar{\psi}_{1} \gamma^{a_{1}} \cdots \gamma^{a_{n}} \psi_{2}=(-)^{n} \bar{\psi}_{2} \gamma^{a_{n}} \cdots \gamma^{a_{1}} \psi_{1} .
\end{aligned}
$$

\section{APPENDIX B: SUPERSPACE OF SUPER-YANG-MILLS THEORY COUPLED TO 2D SUPERGRAVITY}

Up to our knowledge, there is no reference constructing the superspace formulation of 2D $N=1$ supergravity coupled to $N=1$ super-Yang-Mills theory. Thus, in this appendix, we will present the detailed solutions of the Binachi identity (2.19) or (2.20) and discuss the supersymmetric Yang-Mills theory coupled 2D supergravity in the context of superspace formalism. 
In order to solve the super-Yang-Mills Bianchi identities we need the results of the supergravity Bianchi identities (2.12) subject to the constraints (2.21) due to Howe [16]:

$$
\begin{aligned}
& T_{\alpha a}{ }^{b}=-T_{a \alpha}{ }^{b}=0, \\
& T_{a \alpha}{ }^{\beta}=-T_{\alpha a}{ }^{\beta}=\frac{1}{4}\left(\gamma_{a}\right)_{\alpha}{ }^{\beta} S, \\
& T_{a b}{ }^{\alpha}=\frac{i}{4} \epsilon_{a b}\left(\gamma_{5}\right)^{\alpha \beta} D_{\beta} S,
\end{aligned}
$$

where $S$ is the curvature superfield in the Eq.(2.24). We write down the Bianchi identities (2.20) in component form:

$$
\begin{gathered}
\Delta_{[a} F_{b c]}-T_{[a b}^{\alpha} F_{c] \alpha}=0, \\
\Delta_{(\alpha} F_{\beta \gamma)}-2 i \gamma_{(\alpha \beta}^{a} F_{\gamma) a}=0, \\
2 \Delta_{[a} F_{b] \alpha}+\Delta_{\alpha} F_{a b}-2 T_{\alpha[a}^{\beta} F_{b] \beta}+T_{a b}^{\beta} F_{\alpha \beta}=0, \\
2 \Delta_{(\alpha} F_{\beta) a}+\Delta_{a} F_{\alpha \beta}+2 T_{a(\alpha}{ }^{\gamma} F_{\beta) \gamma}-T_{\alpha \beta}{ }^{b} F_{a b}=0 .
\end{gathered}
$$

From Eq.(B3), one may obtain the relation

$$
\begin{aligned}
F_{a \alpha} & =-\frac{i}{2} \gamma_{a}^{\beta \gamma} \Delta_{\beta} F_{\gamma \alpha}-\left(\gamma^{b} \gamma_{a}\right)_{\alpha}{ }^{\beta} F_{b \beta} \\
& =-\frac{i}{6} \gamma_{a}^{\beta \gamma} \Delta_{\beta} F_{\gamma \alpha}+\frac{1}{3}\left(\gamma_{a} \gamma^{b}\right)_{\alpha}{ }^{\beta} F_{b \beta} \\
& \equiv \gamma_{a \alpha}{ }^{\beta} W_{\beta} .
\end{aligned}
$$

It follows from Eq.(2.30) and the 2D identity, $\gamma^{a} \gamma^{b} \gamma_{a}=0$ that $W_{\alpha}$ is of the form

$$
W_{\alpha}=-\frac{i}{2}\left(\gamma_{5}\right)_{\alpha}^{\beta} \Delta_{\beta} W(z)
$$

Thus we obtain

$$
F_{a \alpha}=\frac{i}{2}\left(\gamma_{5} \gamma_{a}\right)_{\alpha}^{\beta} \Delta_{\beta} W(z)
$$

Using the above results, the Eq.(B5) leads to 


$$
F_{a b}=\epsilon_{a b} \frac{1}{4}\left(-\Delta^{2} W+i W S\right)
$$

It is also straightforward to obtain the following relations from the Eqs.(B2) and (BB3):

$$
\begin{gathered}
\Delta_{(a} F_{b c)}=0 \\
\Delta_{\alpha}\left(\epsilon^{a b} F_{a b}+\frac{i}{2} W S\right)+i\left(\gamma^{a}\right)_{\alpha}{ }^{\beta} \Delta_{a} \Delta_{\beta} W=0 .
\end{gathered}
$$

The first equation gives the usual Bianchi identity with respect to the Yang-Mills field strengths $F_{a b}$.

In summary, the SYM Bianchi identities can be completely solved together with the supertorsion components and the field strength $F_{B C}$ can be represented by the scalar superfield $W$ which is defined in terms of the spinor potential $A_{\alpha}$.

For reader's reference, we would like to present some sets of the inverse superzweibein $E_{A}{ }^{M}$ which are frequently used through this paper

$$
\begin{aligned}
& E_{\alpha}{ }^{m}=i\left(\gamma^{m}\right)_{\alpha}{ }^{\mu} \theta_{\mu}-\frac{1}{4} \bar{\theta} \theta\left(\gamma^{n} \gamma^{m}\right)_{\alpha}{ }^{\mu} \chi_{n \mu} \\
& E_{\alpha}{ }^{\mu}=\delta_{\alpha}{ }^{\mu}+\frac{i}{2} \theta^{\nu}\left(\gamma^{m}\right)_{\nu \alpha} \chi_{m}{ }^{\mu}-\frac{i}{4} \bar{\theta} \theta\left\{\left(\gamma_{5} \gamma^{m}\right)_{\alpha}{ }^{\mu} \omega_{m}+\frac{i}{2}\left(\gamma^{n} \gamma^{m}\right)_{\alpha}{ }^{\nu} \chi_{n \nu} \chi_{m}{ }^{\mu}+\frac{1}{2} \delta_{\alpha}{ }^{\mu} A\right\}
\end{aligned}
$$

Under the gauge transformation (2.14) on $A_{\alpha}$, the component fields $\eta$ and $n$ shift by the gauge transformations

$$
\begin{aligned}
& \delta_{X} \eta=i \zeta+i g_{Y M}[\eta, \omega] \\
& \delta_{X} n=i \rho+i g_{Y M}[n, \omega]+\frac{1}{2}(\bar{\eta} \zeta+\bar{\zeta} \eta),
\end{aligned}
$$

so that the fields $\eta$ and $n$ can be completely gauged away. In this Wess-Zumino (WZ) gauge, $\eta=n=0$, the gauge transformations (2.33) of the component fields $v_{m}, \phi$, and $\lambda$ reduce to the ordinary gauge transformations:

$$
\begin{aligned}
& \delta_{\omega} v_{m}=\nabla_{m} \omega, \\
& \delta_{\omega} \phi=i g_{Y M}[\phi, \omega], \\
& \delta_{\omega} \lambda=i g_{Y M}[\lambda, \omega] .
\end{aligned}
$$


On the other hand, the supersymmetry transformation, (2.34), of component fields can be directly calculated by using the Eq. (2.27)

$$
\begin{aligned}
\delta \eta & =i \gamma^{m} \alpha g_{m}+i \gamma_{5} \alpha \phi, \\
\delta n & =-\frac{1}{2} \bar{\alpha} b+\frac{1}{4} \bar{\alpha} \gamma^{m} \gamma^{n} \chi_{m} g_{n}-\frac{1}{4} \bar{\alpha} \gamma^{m} \chi_{m} \phi, \\
\delta g_{m} & =\frac{i}{2} \bar{\alpha} \gamma_{m} b+\frac{i}{2} \bar{\alpha} \gamma^{n} \chi_{m} g_{n}+\frac{i}{4} \bar{\alpha} \gamma_{m} \gamma^{p} \gamma^{n} \chi_{p} g_{n}-\frac{i}{4} \bar{\alpha} \gamma_{5} \gamma^{n} \gamma_{m} \chi_{n} \phi, \\
\delta \phi & =\frac{i}{2} \bar{\alpha} \gamma_{5} b++\frac{i}{4} \bar{\alpha} \gamma_{5} \gamma^{m} \gamma^{n} \chi_{m} g_{n}-\frac{i}{4} \bar{\alpha} \gamma^{m} \chi_{m} \phi, \\
\delta b & =\gamma^{n} \gamma^{m} \alpha \mathcal{D}_{m} g_{n}+\gamma_{5} \gamma^{m} \alpha \partial_{m} \phi-\frac{i}{2} \bar{\alpha} \gamma^{m} \chi_{m} \phi-\frac{i}{4} \gamma^{n} \chi_{m} \bar{\alpha} \gamma^{p} \gamma^{m} \chi_{p} g_{n} \\
& -\frac{i}{4} \gamma_{5} \chi_{m} \bar{\alpha} \gamma^{n} \gamma^{m} \chi_{n} \phi+\frac{1}{4} \gamma^{m} \alpha g_{m} A-\frac{1}{4} \gamma_{5} \alpha \phi A,
\end{aligned}
$$

where $\mathcal{D}_{m} g_{n}=e_{n a} \mathcal{D}_{m} g^{a}$. The above supersymmetry transformations violate the WZ gauge condition $\eta=n=0$. We therefore need to make compensating gauge transformation to maintain the WZ gauge. This is achieved by choosing the gauge parameters as

$$
\begin{aligned}
\zeta & =-\gamma^{m} \alpha v_{m}-\gamma_{5} \alpha \phi \\
n & =-\frac{i}{2} \bar{\alpha} \lambda+\frac{i}{2} \bar{\alpha} \gamma^{m} \gamma^{n} \chi_{m} v_{n}-\frac{i}{4} \bar{\alpha} \gamma^{m} \chi_{m} \phi .
\end{aligned}
$$

From Eq.(B13), we can obtain the covariantized supersymmetry transformations (2.37) for the Yang-Mills multiplet $\left(v_{m}, \phi, \lambda\right)$ by straightforward calculation. In the calculation of the transformation $\delta \lambda$, we have used the following useful identities with respect to the helicity decomposition of the gravitino $\chi_{m}$ :

$$
\begin{aligned}
& \chi_{m}=\tilde{\chi}_{m}+\gamma_{m} \chi, \quad \gamma^{m} \tilde{\chi}_{m}=0, \\
& \gamma_{m} \tilde{\chi}_{n}=\gamma_{n} \tilde{\chi}_{m}, \quad \gamma_{5} \tilde{\chi}_{m}=-e \epsilon_{m n} \tilde{\chi}^{n}, \\
& 2 \overline{\tilde{\chi}}_{m} \tilde{\chi}_{n}=g_{m n} \overline{\tilde{\chi}} \cdot \tilde{\chi}, \quad \overline{\tilde{\chi}}_{m} \gamma_{n} \tilde{\chi}_{p}=0 .
\end{aligned}
$$

In the WZ gauge, the scalar field strength $W(z)$, (2.31), can be calculated straightforwardly by using the Eqs.(2.23), (2.36), and (B12) and the result is

$$
\begin{aligned}
W(z) & =-2 i \phi+2 \bar{\theta} \gamma_{5} \lambda-\frac{1}{2} \bar{\theta} \gamma^{m} \chi_{m} \phi \\
& -\frac{1}{2} \bar{\theta} \theta\left\{\frac{\epsilon^{m n}}{e} v_{m n}+i \bar{\lambda} \gamma_{5} \gamma^{m} \chi_{m}-\frac{i}{4} \bar{\chi}_{n} \gamma^{m} \gamma^{n} \chi_{m} \phi+A \phi\right\} .
\end{aligned}
$$


It is now straightforward although somewhat tedious to calculate $\nabla_{\alpha} W, \nabla_{\alpha} W \nabla^{\alpha} W$ and $E \nabla_{\alpha} W \nabla^{\alpha} W$ from the Eq.(B15) to obtain the super-Yang-Mills action (3.5). 


\section{REFERENCES}

[1] C. M. Hull and P. K. Townsend, Nucl. Phys. B438 (1995) 109, hep-th/9410167.

[2] E. Witten, Nucl. Phys. B443 (1995) 85, hep-th/9503124.

[3] J. H. Schwarz, Lectures on Superstring and M Theory Dualities, Nucl. Phys. Proc. Suppl. 55B (1997) 1, hep-th/9607201 and references therein.

[4] T. Banks, W. Fischler, S. Shenker, and L. Susskind, Phys. Rev. D55 (1997) 5112, hep-th/9610043

[5] E. Witten, Nucl. Phys. B460 (1996) 335, hep-th/9510135.

[6] For reviews of Matrix theory, A. Bilal, M(atrix) Theory: a Pedagogical introduction, hep-th/9710136; T. Banks, Matrix Theory, hep-th/9710231; D. Bigatti and L. Susskind, Reviews of Matrix Theory, hep-th/9712072; W. Taylor IV, Lectures on D-branes, Gauge Theory and M(atrices), hep-th/9801182.

[7] L. Motl, Proposals on nonperturbative superstring interactions, hep-th/9701025; T. Banks and N. Seiberg, Nucl. Phys. B497 (1997) 41, hep-th/9702187.

[8] R. Dijkgraaf, E. Verlinde, and H. Verlinde, Nucl. Phys. B500 (1997) 43, hep-th/9703030; Notes on Matrix and Micro Strings, hep-th/9709107.

[9] M. B. Green and J. H. Schwarz, Phys. Lett. 136B (1984) 367.

[10] P. Ramond, Phys. Rev. D3 (1971) 2415; A. Neveu and J. H. Schwarz, Nucl. Phys. B31 (1971) 86 .

[11] M. Li and T. Yoneya, Phys. Rev. Lett. 78 (1997) 1219, hep-th/9611072; T. Yoneya, Prog. Theor. Phys. 97 (1997) 949, hep-th/9703078.

[12] J. Polchinski, Phys. Rev. Lett. 75 (1995) 4724, hep-th/9510017; TASI Lectures on D-branes, hep-th/9611050. 
[13] L Dixon, J. Harvey, C. Vafa, and E. Witten, Nucl. Phys. B261 (1985) 620; ibid. B274 (1986) 285; L. Dixon, D. Friedan, E. Matinec, and S. Shenker, ibid. B282 (1987) 13; M. Bershadsky and A. Radul, Int. J. Mod. Phys. A2 (1987) 165.

[14] A. M. Polyakov, Gauge Fields and Strings (Harwood Academic Publishers, 1987).

[15] J. Wess and J. Bagger, Supersymmetry and Supergravity, 2nd ed. (Princeton Univ. Press, 1992).

[16] P. Howe, J. Phys. A12: Math. Gen. (1979) 393.

[17] K. Hamada and M. Takao, Phys. Lett. 210B (1988) 120.

[18] Y. Matsumura, N. Sakai, and T. Sakai, Phys. Rev. D52 (1995) 2446, hep-th/9504150.

[19] G. 't Hooft, Nucl. Phys. B75 (1974) 461.

[20] L. Brink, P. Di Vecchia, and P. Howe, Phys. Lett. 65B (1976) 471; S. Deser and B. Zumino, ibid. 65B (1976) 369.

[21] A. M. Polyakov, Phys. Lett. 103B (1981) 207; ibid. 103B (1981) 211.

[22] U. Danielson, G. Ferreti, and B. Sundberg, Int. J. Mod. Phys. A11 (1996) 5463, hep-th/9603081; D. Kabat and P. Pouliot, Phys. Rev. Lett. 77 (1996) 1004, hep-th/9603127; M. R. Douglas, D. Kabat, P. Pouliot, and S. Shenker, Nucl. Phys. B485 (1997) 85, hep-th/9608024.

[23] W. Taylor IV, Phys. Lett. 394B (1996) 283, hep-th/9611042.

[24] R. Dijkgraaf, G. Moore, E. Verlinde, and H. Verlinde, Comm. Math. Phys. 185 (1997) 197, hep-th/9608096.

[25] F. Gliozzi, J. Scherk, and D. Olive, Nucl. Phys. B122 (1977) 253.

[26] M. Green, J. H. Schwarz, and E. Witten, Superstring Theory (Princeton Univ. Press, 1987); D. Lüst and S. Theisen, Lectures on String Theory (Springer-Verlag, 1989). 
[27] L. Susskind, Another Conjecture about M(atrix) Theory, hep-th/9704080; D. Bigatti and L. Susskind, A note on discrete light cone quantization, hep-th/9711063.

[28] P. Goddard, A. Kent, and D. Olive, Phys. Lett. 152B (1985) 88; Comm. Math. Phys. 103 (1986) 105; P. Goddard, W. Nahm, and D. Olive, Phys. Lett. 160B (1985) 111.

[29] E. Witten, Comm. Math. Phys. 92 (1984) 455.

[30] S. J. Gates, C. M. Hull, and M. Rocek, Nucl. Phys. B248 (1984) 157; T. L. Curtright and C. K. Zachos, Phys. Rev. Lett. 53 (1984) 1799; P. Howe and G. Sierra, Phys. Lett. 148B (1984) 451.

[31] V. Periwal and O. Tafjord, Matrix string interactions, hep-th/9802034.

[32] L. P. Kadanoff and A. C. Brown, Ann. Phys. 121 (1979) 318; S.-K. Yang, Nucl. Phys. B285 [FS 19] (1987) 183; H. Saleur, J. Phys. A20 (1987) L1127.

[33] R. Dijkgraaf, E. Verlinde, and H. Verlinde, Comm. Math. Phys. 115 (1988) 649 and references therein. 